\title{
Minimal ideals of group algebras
}

\author{
by \\ David Alexander and Jean Ludwig (Metz)
}

\begin{abstract}
We first study the behavior of weights on a simply connected nilpotent Lie group $G$. Then for a subalgebra $A$ of $L^{1}(G)$ containing the Schwartz algebra $\mathcal{S}(G)$ as a dense subspace, we characterize all closed two-sided ideals of $A$ whose hull reduces to one point which is a character.
\end{abstract}

Introduction. Let $G$ be a simply connected nilpotent Lie group, $\mathfrak{g}$ its Lie algebra, and $A$ a subalgebra of $L^{1}(G)$. To every character $\chi_{l}$ of $A$ we will associate a finite-dimensional translation invariant subspace $\mathcal{P}_{l}$ of the vector space $\mathcal{P}(G)$ of complex polynomials on $G$ and we will show that the set of closed two-sided ideals of $A$ with hull $\left\{\operatorname{Ker} \chi_{l}\right\}$ is in bijection with the set of nonzero translation-invariant subspaces of $\mathcal{P}_{l}$. As an example of $A$ we can take the weighted algebra $L_{w}^{1}(G)$ where $w$ is a weight with polynomial growth. Such weights appear in a natural way in the following manner: let $\pi$ be a unitary continuous irreducible representation of $G$ in a Hilbert space $\mathcal{H}_{\pi}$. We denote by $\mathcal{U}(\mathfrak{g})$ the enveloping algebra of $\mathfrak{g}$. Fix a nonzero integer $k$ and denote by $\mathcal{U}(\mathfrak{g})_{k}$ the vector space generated by the elements of $\mathcal{U}(\mathfrak{g})$ with degree less than $k$. Let $\mathcal{H}_{\pi}^{(k)}$ be the space of $k$ times differentiable vectors in $\mathcal{H}_{\pi}$, i.e.

$$
\mathcal{H}_{\pi}^{(k)}=\left\{\xi \in \mathcal{H}_{\pi} \mid \forall z \in \mathcal{U}(\mathfrak{g})_{k}: d \pi(z) \xi \in \mathcal{H}_{\pi}\right\} .
$$

Fix a basis $\left(z^{i}\right)_{|i| \leq k}$ of $\mathcal{U}(\mathfrak{g})_{k}$. We equip $\mathcal{H}_{\pi}^{(k)}$ with the norm

$$
\|\xi\|_{k}=\left(\sum_{|i| \leq k}\left\|d \pi\left(z^{i}\right) \xi\right\|^{2}\right)^{1 / 2} .
$$

The space $\mathcal{H}_{\pi}^{(k)}$ with this norm is complete. Denoting by $\|\pi(x)\|_{\text {op }}$ the norm of the operator $\pi(x): \mathcal{H}_{\pi}^{(k)} \rightarrow \mathcal{H}_{\pi}^{(k)}$, we then have

$$
\|\pi(x)\|_{\mathrm{op}} \leq\left\|\left.\operatorname{Ad}(x)\right|_{\mathcal{U}(\mathfrak{g})_{k}}\right\|_{\mathrm{HS}}
$$

2000 Mathematics Subject Classification: Primary 43A20. 
where \|\|$_{\text {HS }}$ denotes the Hilbert-Schmidt norm. Denote by $w^{(k)}(x)$ the right side of this inequality. The function $x \mapsto w^{(k)}(x)$ is a natural example of a weight on $G$ attached to $\pi$. By a weight on a topological group $G$, we mean a measurable function $w$ on $G$ with values in $[1,+\infty[$ such that for all $s$ and $t$ in $G$,

$$
w(s t) \leq w(s) w(t) .
$$

The preceding result leads naturally to the study of weights on nilpotent Lie groups. The first section will give another example of a natural weight. Other examples of weights come from Banach space representations of topological groups. Let $X$ be a Banach space and let $(T, X)$ be a Banach space representation of $G$ on $X$. That means that for every $s$ in $G$, we have a bounded invertible operator $T(s)$ on $X$ such that the mapping $s \mapsto T(s)$ is a homomorphism of groups and the mappings $s \mapsto T(s) x$ are continuous for every $x$ in $X$. Then the operator norm $\|T(s)\|_{\text {op }}$ is a measurable function on $G$ and defines a symmetric weight $w_{T}: s \mapsto \max \left(\|T(s)\|_{\mathrm{op}},\left\|T\left(s^{-1}\right)\right\|_{\mathrm{op}}\right)$.

Take for example the 3-dimensional Heisenberg group $G=H_{1}$. For $x$ in $H_{1}$ write $x=(a, b, t)$ and let $(X, Y, Z)$ be a basis of the Lie algebra $\mathfrak{h}_{1}$ of $H_{1}$ with $[X, Y]=Z$. We have

$$
\operatorname{Ad}(x) X=X-b Z, \quad \operatorname{Ad}(x) Y=Y+a Z, \quad \operatorname{Ad}(x) Z=Z .
$$

After an easy computation, we find

$$
w^{(2)}(x)=\left(9+7 a^{2}+7 b^{2}+a^{2} b^{2}+a^{4}+b^{4}\right)^{1 / 2}
$$

and

$$
\frac{1}{\sqrt{2}}\left(1+a^{2}+b^{2}\right) \leq w^{(2)}(x) \leq 3\left(1+a^{2}+b^{2}\right) .
$$

\section{Weights on topological (in particular nilpotent Lie) groups.} Weights allow us to define Banach subalgebras of $L^{1}(G)$, the so-called Beurling algebras. This section studies the growth of the "most natural" weight attached to a connected locally compact group. This weight is of importance because it dominates all common weights. We end this section with a restriction property of this weight.

Definition. Let $G$ be a topological group and $S$ a subset of $G$. We write $S^{0}=\{e\}$ and for all $n$ in $\mathbb{N}^{*}$,

$$
S^{n}=\left\{s_{1} \ldots s_{n} \mid s_{i} \in S\right\} .
$$

When $G$ is locally compact, for $s$ in $G$, we denote by $\mathfrak{V}_{G}(s)$ the set of compact neighborhoods of $s$ in $G$.

In the following proposition we recall the "most natural" weight attached to a connected locally compact group as in [9]. 
1.1. Proposition. Let $G$ be a connected locally compact group and $U$ an element of $\mathfrak{V}_{G}(e)$. Then $G=\bigcup_{n \in \mathbb{N}} U^{n}$ and the map $\tau_{U}: G \rightarrow \mathbb{N}$ defined by

$$
\tau_{U}(s)=\min \left\{n \in \mathbb{N} \mid s \in U^{n}\right\}
$$

is measurable and satisfies

$$
\tau_{U}(s)=0 \Leftrightarrow s=e, \quad \tau_{U}(s t) \leq \tau_{U}(s)+\tau_{U}(t) .
$$

If in addition $U$ is symmetric, then

$$
\tau_{U}\left(s^{-1}\right)=\tau_{U}(s)
$$

It seems difficult to define canonically the notion of a "polynomial function" on any group $G$. In the absence of such a notion, the following definition tries to define in a natural way a function "of polynomial growth" on a class of groups as large as possible.

1.2. Definition. Let $G$ be a connected locally compact group. A function $f: G \rightarrow \mathbb{C}$ is said to be of polynomial growth if for all $U$ in $\mathfrak{V}_{G}(e)$, there exists a polynomial $P_{U}$ in one variable, with real coefficients, such that for all $s$ in $G$,

$$
|f(s)| \leq P_{U}\left(\tau_{U}(s)\right) .
$$

For example for a connected compact group $G$, the functions with polynomial growth on $G$ are bounded functions. More generally, it is easy to check that under the conditions of 1.2, a function with polynomial growth is bounded on all compact subsets. Since for any two elements $U$ and $V$ of $\mathfrak{V}_{G}(e)$, there exist strictly positive numbers $k$ and $k^{\prime}$ such that

$$
\tau_{V} \leq k \tau_{U} \leq k^{\prime} \tau_{V}
$$

it follows that if $f: G \rightarrow \mathbb{C}$ satisfies $|f| \leq P_{U} \circ \tau_{U}$ for one compact neighborhood $U$ of $e$ in $G$, then such a relation is true for all compact neighborhoods of $e$ in $G$, i.e. $f$ is of polynomial growth.

Notation. Let $G$ be a group. For $f: G \rightarrow \mathbb{C}$, we denote by $\check{f}$ the function $s \mapsto f\left(s^{-1}\right)$.

It is clear that the set of weights on $G$ is stable under pointwise multiplication, involution $w \mapsto \check{w}$, finite simple limit, finite upper hull, and left composition by functions of the form $\exp \circ f \circ \ln$, where $f$ is an increasing and subadditive function $\mathbb{R}_{+} \rightarrow \mathbb{R}_{+}$. Such functions are studied in [8].

1.3. ExAmple. For a connected locally compact group $G$ and $U$ in $\mathfrak{V}_{G}(e)$, the map $1+\tau_{U}$, denoted by $w_{U}$, is clearly a weight on $G$, satisfying in addition

$$
w_{U}(s t) \leq w_{U}(s)+w_{U}(t) .
$$

This weight will be studied in detail in the following when $G$ will be assumed to be a nilpotent Lie group. By [6], we have: 
1.4. Proposition. Let $G$ be a connected Lie group with Lie algebra $\mathfrak{g}$. $A$ norm \|\| on the vector space $\mathfrak{g}$ being fixed, for all $U$ in $\mathfrak{V}_{G}(e)$, there exists a strictly positive number $c_{U}$ such that for all $X$ in $\mathfrak{g}$,

$$
w_{U}(\exp X)<2+c_{U}\|X\| \text {. }
$$

Until the end of this section, $G$ denotes a simply connected nilpotent Lie group with Lie algebra $\mathfrak{g}$. Starting with $\mathfrak{g}_{0}=\mathfrak{g}$, we define $\mathfrak{g}_{m}$ for $m$ in $\mathbb{N}^{*}$ as the real vector space generated by the set of $[X, Y]$ where $X$ runs through $\mathfrak{g}$ and $Y$ runs through $\mathfrak{g}_{m-1}$. The step of nilpotency of $\mathfrak{g}$ is denoted by $n$; this means that $\mathfrak{g}_{n}$ reduces to $\{0\}$ and $\mathfrak{g}_{n-1}$ is nonzero. Hence, an element $X$ of $\mathfrak{g}$ belongs to $\mathfrak{g}_{i}$ if and only if $X$ is a linear combination of terms requiring at least $i$ brackets in all. For all $i$ in $\{1, \ldots, n\}$, choose a complementary subspace $V_{i}$ of $\mathfrak{g}_{i}$ in $\mathfrak{g}_{i-1}$. Then

$$
\mathfrak{g}=\bigoplus_{i=1}^{n} V_{i} .
$$

For all $k$ in $\{0, \ldots, n\}$, let $G_{k}$ be $\exp \mathfrak{g}_{k}$. Then $G_{k}$ is the closure in $G$ of the subgroup generated by the elements $x y x^{-1} y^{-1}$ where $x$ runs through $G$ and $y$ runs through $G_{k-1}$. The exponential map exp is a $C^{\infty}$ diffeomorphism of $\mathfrak{g}$ onto $G$, which allows us to identify $G$ with the real vector space $\mathfrak{g}$ as manifolds. If $\mathfrak{g}$ is endowed with the Baker-Campbell-Hausdorff product

$$
\begin{aligned}
X \cdot Y= & X+Y+\frac{1}{2}[X, Y]+\frac{1}{12}([X,[X, Y]]+[Y,[Y, X]]) \\
& +(\text { commutators of order } 3 \text { at least })
\end{aligned}
$$

then exp is an isomorphism of topological groups from $\mathfrak{g}$ onto $G$, which allows us to identify the groups $G$ and $(\mathfrak{g}, \cdot)$. For this group law, $-X$ is the inverse of $X$. Finally, for $X$ and $Y$ in $\mathfrak{g}$, we set

$$
\{X, Y\}=X \cdot Y \cdot(-X) \cdot(-Y) \text {. }
$$

By [21], we have:

1.5. Lemma. Let $\mathfrak{g}$ be a nilpotent Lie algebra of step $n$. For all $X_{1}, \ldots$ $\ldots, X_{n}$ in $\mathfrak{g}$, we have

$$
\begin{aligned}
{\left[X_{1},\left[X_{2},\left[\ldots, X_{n-1}\right] \ldots\right]\right] } & \equiv\left\{X_{1},\left\{X_{2},\left\{\ldots, X_{n-1}\right\} \ldots\right\}\right\} \bmod \mathfrak{g}_{n-1}, \\
{\left[X_{1},\left[X_{2},\left[\ldots, X_{n}\right] \ldots\right]\right] } & =\left\{X_{1},\left\{X_{2},\left\{\ldots, X_{n}\right\} \ldots\right\}\right\} .
\end{aligned}
$$

In the following proposition, the bracket of two elements $X$ and $Y$ will be written as product in the group $\mathfrak{g}$ of $a_{i} X$ and $b_{i} Y$ where $a_{i}$ and $b_{i}$ are real numbers. We give a bound for the number of factors in the product, which improves a result of [21].

1.6. Proposition. Let $\mathfrak{g}$ be a nilpotent Lie algebra of step $n$ greater than 2 . 
1) There exists an integer $m$, depending only on $n$ and $2 m$ real numbers $a_{1}, \ldots, a_{m}, b_{1}, \ldots, b_{m}$, such that for all $X$ and $Y$ in $\mathfrak{g}$, we have

$$
X+Y=\prod_{i=1}^{m}\left(a_{i} X\right) \cdot\left(b_{i} Y\right) .
$$

2) There exists an integer $p$, depending only on $n$ and $2 p$ real numbers $c_{1}, \ldots, c_{p}, d_{1}, \ldots, d_{p}$, such that for all $X$ and $Y$ in $\mathfrak{g}$, we have

$$
[X, Y]=\prod_{i=1}^{p}\left(c_{i} X\right) \cdot\left(d_{i} Y\right)
$$

In addition $m$ and $p$ are less than $2^{n}\left(2^{n}-5\right)+2 n+2$.

Proof. 1) If $n=2$, then for all $X$ and $Y$ in $\mathfrak{g}$, we have

$$
X+Y=\frac{X}{2} \cdot Y \cdot \frac{X}{2} .
$$

Assume the result is true for a nilpotent Lie algebra of step $n-1 \geq 2$ and let $\mathfrak{g}$ be a nilpotent Lie algebra of step $n$. Since $\mathfrak{g} / \mathfrak{g}_{n-1}$ is nilpotent of step $n-1$, for all $X_{1}$ and $X_{2}$ in $\mathfrak{g}$, we have by the induction hypothesis

$$
X_{1}+X_{2}=\prod_{i=1}^{m}\left(c_{i} X_{1}\right) \cdot\left(d_{i} X_{2}\right)+u\left(X_{1}, X_{2}\right)
$$

where $u\left(X_{1}, X_{2}\right)$ belongs to $\mathfrak{g}_{n-1}$, hence to the center of $\mathfrak{g}$. There exist real numbers $c_{i_{1} \ldots i_{n}}$, where $\left(i_{1}, \ldots, i_{n}\right)$ runs through $\{1,2\}^{n}$, such that

$$
\begin{aligned}
u\left(X_{1}, X_{2}\right) & =\sum_{\left(i_{1}, \ldots, i_{n}\right) \in\{1,2\}^{n}} c_{i_{1} \ldots i_{n}}\left[X_{i_{1}},\left[X_{i_{2}},\left[\ldots, X_{i_{n}}\right] \ldots\right]\right] \\
& =\prod_{\left(i_{1}, \ldots, i_{n}\right) \in\{1,2\}^{n}}\left[c_{i_{1} \ldots i_{n}} X_{i_{1}},\left[X_{i_{2}},\left[\ldots, X_{i_{n}}\right] \ldots\right]\right] .
\end{aligned}
$$

By Lemma 1.5, we have

$$
u\left(X_{1}, X_{2}\right)=\prod_{\left(i_{1}, \ldots, i_{n}\right) \in\{1,2\}^{n}}\left\{c_{i_{1} \ldots i_{n}} X_{i_{1}},\left\{X_{i_{2}},\left\{\ldots, X_{i_{n}}\right\} \ldots\right\}\right\}
$$

and then

$$
X_{1}+X_{2}=\prod_{i=1}^{m}\left(c_{i} X_{1}\right) \cdot\left(d_{i} X_{2}\right) \cdot \prod_{\left(i_{1}, \ldots, i_{n}\right) \in\{1,2\}^{n}}\left\{c_{i_{1} \ldots i_{n}} X_{i_{1}},\left\{X_{i_{2}},\left\{\ldots, X_{i_{n}}\right\} \ldots\right\}\right\}
$$

where $c_{1 \ldots 1}$ and $c_{2 \ldots 2}$ are zero. Denoting by $m_{n}$ the number of factors sufficient to write $X_{1}+X_{2}$ as a product when $\mathfrak{g}$ is nilpotent of step $n$, we have shown that $m_{2}=3$; we can check that

$$
m_{n}=m_{n-1}+\left(2^{n}-2\right)\left(3 \cdot 2^{n-1}-2\right)
$$


and consequently

$$
m_{n}=2^{n+1}\left(2^{n}-5\right)+4 n+3 .
$$

2) Let us prove the second assertion. If $n=2$, then for all $X$ and $Y$ in $\mathfrak{g}$, we have

$$
[X, Y]=X \cdot Y \cdot(-X) \cdot(-Y) .
$$

The proof of the rest of the assertion is similar and we find that

$$
p_{n}=2^{n+1}\left(2^{n}-5\right)+4 n+4
$$

where $p_{n}$ indicates the number of factors sufficient to write $\left[X_{1}, X_{2}\right]$ as a product when $\mathfrak{g}$ is nilpotent of step $n$.

1.7. Corollary. Let $\mathfrak{g}$ be a nilpotent Lie algebra of step $n$ greater than 2. Let $X_{1}, \ldots, X_{p}$ be elements of $\mathfrak{g}$ of the form

$$
X_{i}=\left[X_{i}^{1},\left[X_{i}^{2},\left[\ldots, X_{i}^{k_{i}}\right] \ldots\right]\right] .
$$

Then there exists an integer $q$, depending only on $p$ and $n$, such that

$$
\sum_{i=1}^{p} X_{i}=\prod_{j=1}^{q} \prod_{\substack{1 \leq i_{j} \leq p \\ 1 \leq l_{i_{j}} \leq k_{i_{j}}}} c_{i_{j} l_{i_{j}}} X_{i_{j}}^{l_{i_{j}}} .
$$

Proof. It suffices to apply the previous proposition as many times as necessary.

We recall that $G$ denotes a simply connected nilpotent Lie group with Lie algebra $\mathfrak{g}$. In the rest of this section fix a euclidean norm \|\| on $\mathfrak{g}$, and denote by $U$ the unit ball $B$ of $\mathfrak{g}$.

1.8. COROLlary. There exists a real number $c_{1}$ such that for all $j$ in $\{1, \ldots, n-1\}$, and $X$ in $\mathfrak{g}_{j}$,

$$
w_{U}(X) \leq c_{1}(1+\|X\|)^{1 / j+1}
$$

Proof. Let $j$ be in $\{1, \ldots, n-1\}$ and fix a basis $\left(X_{1}, \ldots, X_{p}\right)$ of $\mathfrak{g}_{j}$. Each $X_{i}$ can be chosen such that

$$
X_{i}=\left[X_{i}^{1},\left[X_{i}^{2},\left[\ldots, X_{i}^{j+1}\right] \ldots\right]\right]
$$

for certain vectors $X_{i}^{k}$. Let $X$ be in $\mathfrak{g}_{j}$. We can write

$$
(1+\|X\|)^{-1} X=\sum_{i=1}^{p} c_{i} X_{i}
$$


where $\left|c_{i}\right|<1$, and then

$$
\begin{aligned}
X= & \sum_{i=1}^{p} c_{i}(1+\|X\|)\left[X_{i}^{1},\left[X_{i}^{2},\left[\ldots, X_{i}^{j+1}\right] \ldots\right]\right] \\
= & \sum_{i=1}^{p}\left[c_{i}(1+\|X\|)^{1 / j+1} X_{i}^{1},\right. \\
& {\left.\left[(1+\|X\|)^{1 / j+1} X_{i}^{2},\left[\ldots,(1+\|X\|)^{1 / j+1} X_{i}^{j+1}\right] \ldots\right]\right] . }
\end{aligned}
$$

By Corollary 1.7, it follows that

$$
X=\prod_{m=1}^{q} \prod_{\substack{1 \leq i_{m} \leq p \\ 1 \leq r_{m} \leq j+1}} c_{i_{m} r_{m}}(1+\|X\|)^{1 / j+1} X_{i_{m}}^{r_{m}}
$$

for a certain integer $q$ and some real numbers $c_{i_{m} r_{m}}$, depending only on $j$, $n, p$. Let $s$ be the number of factors in the above product. Put

$$
\begin{aligned}
c & =\max \left\{\left|c_{i_{m} r_{m}}\right| \mid 1 \leq i_{m} \leq p, 1 \leq r_{m} \leq j+1,1 \leq m \leq q\right\} \\
t & =\max \left\{\left\|X_{i}^{k}\right\| \mid 1 \leq i \leq p, 1 \leq k \leq j+1\right\}
\end{aligned}
$$

Hence

$$
X \in U^{s\left(1+E\left(c t(1+\|X\|)^{1 / j+1}\right)\right)}
$$

where $E$ indicates the integer part function, from which, by definition of $w_{U}$,

$$
\begin{aligned}
w_{U}(X) & \leq 1+s\left(1+E\left(\operatorname{ct}(1+\|X\|)^{1 / j+1}\right)\right) \\
& \leq 1+s+\operatorname{sct}(1+\|X\|)^{1 / j+1} \\
& \leq(1+s+s c t)(1+\|X\|)^{1 / j+1}
\end{aligned}
$$

1.9. Proposition. There exists a real number $c_{2}$ such that for all $X$ in $\mathfrak{g}$ and all $j$ in $\{1, \ldots, n\}$ we have

$$
\left(1+\left\|X_{j}\right\|\right)^{1 / j} \leq c_{2} w_{U}(X)
$$

where $X_{j}$ indicates the component of $X$ belonging to $V_{j}$.

Proof. 1) Let $\varepsilon$ be a strictly positive number. Let us show by induction on $m$ that there exists a real number $a_{\varepsilon}=O(\varepsilon)$ such that if $X \in(\varepsilon B)^{m}$, then $\left\|X_{j}\right\|<a_{\varepsilon}(1+m)^{j}$.

If $m=1$, then $\left\|X_{j}\right\|<\varepsilon$, hence we take $a_{\varepsilon}=\varepsilon 2^{-j}$.

Assume the result is true for $m-1$ and let $X$ be in $(\varepsilon B)^{m}$. Then $X$ can be written as $Y \cdot W$ where $Y \in(\varepsilon B)^{m-1}$ and $W \in \varepsilon B$. By the induction hypothesis,

$$
\left\|Y_{j}\right\| \leq a_{\varepsilon} m^{j}
$$


where $a_{\varepsilon}=O(\varepsilon)$. By the Baker-Campbell-Hausdorff formula, we have

$$
(Y \cdot W)_{j}=Y_{j}+W_{j}+Q_{j}(Y, W)
$$

where

$$
Q_{j}(Y, W)=\sum_{\substack{i_{1}, \ldots, i_{p} \geq 1 \\ i_{1}+\ldots+i_{p} \leq j}} c_{i_{1} \ldots i_{p}}^{j}\left[T_{i_{1}},\left[\ldots, T_{i_{p}}\right] \ldots\right]_{j}
$$

and where each $T_{i_{k}}$ is $Y_{i_{k}}$ or $W_{i_{k}}$, i.e. an element of $V_{i_{k}}$. Since each $W_{i_{k}}$ appears at least once in each bracket, it follows that for $\varepsilon$ small enough

$$
\begin{aligned}
\left\|\left[T_{i_{1}},\left[\ldots, T_{i_{p}}\right] \ldots\right]_{j}\right\| & \leq\left\|\left[T_{i_{1}},\left[\ldots, T_{i_{p}}\right] \ldots\right]\right\| \leq \varepsilon\left\|T_{i_{1}}\right\| \ldots\left\|\widehat{T}_{i_{k}}\right\| \ldots\left\|T_{i_{p}}\right\| \\
& \leq \varepsilon a_{\varepsilon} m^{i_{1}} \ldots \widehat{a_{\varepsilon} m^{i_{k}}} \ldots a_{\varepsilon} m^{i_{p}} \leq \varepsilon m^{j-1}
\end{aligned}
$$

and hence, by (3),

where

$$
\left\|Q_{j}(Y, W)\right\| \leq \varepsilon m^{j-1} \sum_{\substack{i_{1}, \ldots, i_{p} \geq 1 \\ i_{1}+\ldots+i_{p} \leq j}}\left|c_{i_{1} \ldots i_{p}}^{j}\right| \leq \varepsilon c N m^{j-1}
$$

$$
c=\max \left\{\left|c_{i_{1} \ldots i_{p}}^{j}\right| \mid i_{1}, \ldots, i_{p} \geq 1 \text { and } i_{1}+\ldots+i_{p} \leq j\right\}
$$

and $N$ is the number of terms in the preceding sum. We then deduce, by (1) and (2), that

$$
\begin{aligned}
\left\|(Y \cdot W)_{j}\right\| & \leq\left\|Y_{j}\right\|+\left\|W_{j}\right\|+\left\|Q_{j}(Y, W)\right\| \\
& \leq a_{\varepsilon} m^{j}+\varepsilon+\varepsilon c N m^{j-1} \leq c_{\varepsilon}(1+m)^{j}
\end{aligned}
$$

where $c_{\varepsilon}=a_{\varepsilon}+\varepsilon+\varepsilon c N$. Finally $\left\|X_{j}\right\| \leq c_{\varepsilon}(1+m)^{j}$ where $c_{\varepsilon}=O(\varepsilon)$. We now choose our new $a_{\varepsilon}$ as $c_{\varepsilon}$.

2) Let $X$ be in $U, \varepsilon$ be a strictly positive number and $M_{\varepsilon}$ the integer such that

$$
M_{\varepsilon}-1<\varepsilon^{-1} \leq M_{\varepsilon}
$$

Then

$$
\left\|M_{\varepsilon}^{-1} X\right\| \leq \varepsilon\|X\| \leq \varepsilon
$$

therefore $M_{\varepsilon}^{-1} X$ belongs to $\varepsilon B$ and consequently $X \in(\varepsilon B)^{M_{\varepsilon}}$.

3) Let $X$ be a nonzero element of $\mathfrak{g}$. Fix $\varepsilon$ small enough so that $a_{\varepsilon}<1$ in 1). By definition, $X$ belongs to $U^{w_{U}(X)-1}$, then by 2) to $(\varepsilon B)^{M_{\varepsilon}\left(w_{U}(X)-1\right)}$, and by 1 ),

$$
\left(1+\left\|X_{j}\right\|\right)^{1 / j} \leq\left(1+M_{\varepsilon}^{j}\right)^{1 / j} w_{U}(X) .
$$

1.10. Proposition. There exists a real number $c_{3}$ such that for all $Y_{1}, \ldots, Y_{n}$ where each $Y_{j}$ belongs to $\mathfrak{g}_{j-1}$, we have

$$
\left\|X_{j}\right\|^{1 / j} \leq c_{3} \max _{1 \leq i \leq j}\left(1+\left\|Y_{i}\right\|\right)^{1 / i}, \quad\left\|Y_{j}\right\|^{1 / j} \leq c_{3} \max _{1 \leq i \leq j}\left(1+\left\|X_{i}\right\|\right)^{1 / i},
$$

where $X_{j}$ indicates the component of $Y_{1} \ldots Y_{n}$ belonging to $V_{j}$. 
Proof. Fix $j$ in $\{1, \ldots, n\}$. By the Baker-Campbell-Hausdorff formula,

$$
X_{j}=Y_{j}+\sum_{\substack{i_{1}, \ldots, i_{p} \geq 1 \\ i_{1}+\ldots+i_{p} \leq j}} c_{i_{1} \ldots i_{p}}^{j}\left[Y_{i_{1}},\left[\ldots, Y_{i_{p}}\right], \ldots\right]_{j},
$$

hence

$$
\begin{aligned}
\left\|X_{j}\right\| & \leq\left\|Y_{j}\right\|+\sum_{\substack{i_{1}, \ldots, i_{p} \geq 1 \\
i_{1}+\ldots+i_{p} \leq j}}\left|c_{i_{1} \ldots i_{p}}^{j}\right|\left\|Y_{i_{1}}\right\| \ldots\left\|Y_{i_{p}}\right\| \\
& \leq\left\|Y_{j}\right\|+c \sum_{\substack{i_{1}, \ldots, i_{p} \geq 1 \\
i_{1}+\ldots+i_{p} \leq j}}\left(\left\|Y_{i_{1}}\right\|^{1 / i_{1}}\right)^{i_{1}} \ldots\left(\left\|Y_{i_{p}}\right\|^{1 / i_{p}}\right)^{i_{p}} \\
& \leq(1+c N)\left(\max _{1 \leq i \leq j}\left(1+\left\|Y_{i}\right\|\right)^{1 / i}\right)^{j}
\end{aligned}
$$

where

$$
c=\max \left\{\left|c_{i_{1} \ldots i_{p}}^{j}\right| \mid i_{1}, \ldots, i_{p} \geq 1 \text { and } i_{1}+\ldots+i_{p} \leq j\right\}
$$

and $N$ is the number of terms in the previous sum. Finally,

$$
\left\|X_{j}\right\|^{1 / j} \leq(1+c N)^{1 / j} \max _{1 \leq i \leq j}\left(1+\left\|Y_{i}\right\|\right)^{1 / i} .
$$

The second relation follows similarly.

By 1.2 all the weights $w_{U}$ are equivalent on a connected group $G$. Hence we fix a compact neighborhood $U$ of $e$ in $G$ and we write $w_{G}$ instead of $w_{U}$. We can then summarize the previous results in the following theorem:

1.11. TheOREM. Let $G$ be a simply connected nilpotent Lie group with Lie algebra $\mathfrak{g}$. Let $\mathfrak{g}=\bigoplus_{i=1}^{n} V_{i}$ where $V_{i} \oplus \mathfrak{g}_{i}=\mathfrak{g}_{i-1}$ and where $\left(\mathfrak{g}_{i}\right)_{0 \leq i \leq n-1}$ is the central decreasing sequence of $\mathfrak{g}$. Then there exist real numbers $c$ and $c^{\prime}$ such that for all $X$ in $\mathfrak{g}$, we have

$$
c \max _{1 \leq i \leq n}\left(1+\left\|X_{i}\right\|\right)^{1 / i} \leq w_{G}(\exp X) \leq c^{\prime} \max _{1 \leq i \leq n}\left(1+\left\|X_{i}\right\|\right)^{1 / i}
$$

where $X_{i}$ indicates the component of $X$ belonging to $V_{i}$.

Proof. Proposition 1.9 shows the existence of $c$. Let now $X$ be in $\mathfrak{g}$. We can find $Y_{1}, \ldots, Y_{n}$, where each $Y_{j}$ belongs to $\mathfrak{g}_{j-1}$, such that $\exp X=$ $\exp Y_{1} \ldots \exp Y_{n}$. Hence, by 1.3, we have

$$
w_{G}(\exp X) \leq \sum_{j=1}^{n} w_{G}\left(\exp Y_{j}\right)
$$

and by Corollary 1.8,

$$
w_{G}(\exp X) \leq c_{1} \sum_{j=1}^{n}\left(1+\left\|Y_{j}\right\|\right)^{1 / j} .
$$


It now follows from Proposition 1.10 that

$$
\begin{aligned}
w_{G}(\exp X) & \leq c_{1} \sum_{j=1}^{n}\left[1+c_{3}^{j} \max _{1 \leq i \leq j}\left(1+\left\|X_{i}\right\|\right)^{j / i}\right]^{1 / j} \\
& \leq c_{1} \sum_{j=1}^{n} 1+c_{3} \max _{1 \leq i \leq j}\left(1+\left\|X_{i}\right\|\right)^{1 / i} \\
& \leq c_{1} n\left(1+c_{3}\right) \max _{1 \leq i \leq n}\left(1+\left\|X_{i}\right\|\right)^{1 / i} .
\end{aligned}
$$

Notation. For all $k$ in $\{1, \ldots, n\}$, the set $U \cap G_{k}$ denoted by $V_{k}$ is a symmetric compact neighborhood of $e$ in $G_{k}$, and the weight $w_{V_{k}}$ on $G_{k}$ defined in 1.3 will be denoted by $w_{G_{k}}$.

1.12. THEOREM. There exists a strictly positive number $c$ such that for all $k$ in $\{1, \ldots, n\}$, we have

$$
\left.w_{G}\right|_{G_{k}} \leq c w_{G_{k}}^{1 / k+1} .
$$

Proof. We easily show by induction on $i$ that for all $i$ in $\mathbb{N}$,

$$
\left(\mathfrak{g}_{k}\right)_{i} \subset \mathfrak{g}_{(k+1)(i+1)-1} .
$$

Denote by $p_{k}$ the step of nilpotency of $\mathfrak{g}_{k}$ and let $Y$ be an element of $\mathfrak{g}_{k}$. Then

$$
Y=Y_{1} \ldots Y_{p_{k}}=X_{1}+\ldots+X_{p_{k}}
$$

where each $Y_{i}$ belongs to $\left(\mathfrak{g}_{k}\right)_{i-1}$ and $X_{i}$ to $\left(V_{k}\right)_{i}$ where, as at the beginning of this section, $\left(\mathfrak{g}_{k}\right)_{i-1}$ is the direct sum of $\left(\mathfrak{g}_{k}\right)_{i}$ and $\left(V_{k}\right)_{i}$ for all $i$ in $\left\{1, \ldots, p_{k}\right\}$. As noticed in 1.3 , we have

$$
w_{G}(\exp Y)=w_{G}\left(\exp Y_{1} \ldots \exp Y_{p_{k}}\right) \leq w_{G}\left(\exp Y_{1}\right)+\ldots+w_{G}\left(\exp Y_{p_{k}}\right) \text {. }
$$

Now each $Y_{i}$ belongs to $\mathfrak{g}_{(k+1) i-1}$ by $(1)$, and so by Corollary 1.8 ,

$$
w_{G}\left(\exp Y_{i}\right) \leq c_{1}\left(1+\left\|Y_{i}\right\|\right)^{1 /(k+1) i} ;
$$

hence

$$
\begin{aligned}
w_{G}(\exp Y) & \leq c_{1} \sum_{i=1}^{p_{k}}\left(1+\left\|Y_{i}\right\|\right)^{1 /(k+1) i} \\
& \leq c_{1} p_{k} \max _{1 \leq i \leq p_{k}}\left(1+\left\|Y_{i}\right\|\right)^{1 /(k+1) i} \\
& \leq c_{1} p_{k}+c_{1} p_{k}\left(\max _{1 \leq i \leq p_{k}}\left\|Y_{i}\right\|^{1 / i}\right)^{1 / k+1} \\
& \leq c_{1} p_{k}+c_{1} p_{k}\left(c_{3} \max _{1 \leq i \leq p_{k}}\left(1+\left\|X_{i}\right\|\right)^{1 / i}\right)^{1 / k+1} \\
& \leq c_{1} p_{k}+c_{1} p_{k}\left(c_{3} c_{2} w_{G_{k}}(\exp Y)\right)^{1 / k+1} \\
& \leq\left(c_{1} p_{k}+c_{1} p_{k}\left(c_{2} c_{3}\right)^{1 / k+1}\right)\left(w_{G_{k}}(\exp Y)\right)^{1 / k+1}
\end{aligned}
$$

where (2) and (3) result from Propositions 1.10 and 1.9 respectively. 
1.13. Corollary. Let $N$ be a subgroup of $G_{1}$ and let $(\pi, X)$ be a Banach space representation of $G$ on $X$. If $\left.\pi\right|_{N}$ is given by $\chi 1_{X}$ for some character $\chi$ of $N$, then $\chi$ must be unitary.

Proof. Assume that $\left.\pi\right|_{N}$ is a (continuous) nonunitary character of $N$. Denote by $\mathfrak{n}$ the Lie algebra of $N$. Let $U$ be in $\mathfrak{V}_{G}(e)$. First, for all $s$ in $G$ distinct from $e$ we have

$$
s=s_{1} \ldots s_{\tau_{U}(s)}
$$

hence

$$
|\pi(s)|=\left|\pi\left(s_{1}\right)\right| \ldots\left|\pi\left(s_{\tau_{U}(s)}\right)\right| \leq e^{k_{U} \tau_{U}(s)} \leq e^{k_{U} w_{G}(s)}
$$

where

$$
e^{k_{U}}=\sup _{s \in U}|\pi(s)|
$$

By hypothesis, there exist two real linear forms $\alpha$ and $\beta$ on $\mathfrak{n}$, with $\alpha \neq 0$, such that

$$
\pi(\exp X)=e^{\langle\alpha+i \beta, X\rangle}, \quad X \in \mathfrak{n} .
$$

Fix $X$ in $\mathfrak{n}$ such that $\langle\alpha, X\rangle=1$. Then for all $t$ in $\mathbb{R}$, from (1) we have

$$
e^{t}=|\pi(\exp (t X))| \leq e^{k_{U} w_{G}(\exp (t X))} .
$$

Let $V=U \cap G_{1}$. By Theorem 1.12

$$
w_{G}(\exp (t X))=\left.w_{G}\right|_{G_{1}}(\exp (t X)) \leq c\left(w_{G_{1}}(\exp (t X))\right)^{1 / 2}
$$

and by Proposition 1.4,

$$
w_{G_{1}}(\exp (t X))<2+c_{V}\|t X\|
$$

hence

$$
e^{t} \leq e^{k_{U} c \sqrt{2+c_{V}|t||X| \mid}}
$$

This last inequality is false for $t$ large enough.

2. Spectral synthesis for nilpotent Lie groups. Let $G$ be a connected Lie group, $\mathfrak{g}$ its Lie algebra, and $\mathfrak{g}^{*}$ the dual vector space of $\mathfrak{g}$. The set of equivalence classes of irreducible continuous unitary representations of $G$ is denoted by $\widehat{G}$. When $G$ is abelian, by Schur's lemma, $\widehat{G}$ is in bijection with the group of continuous characters of $G$ into the multiplicative group $U$ of complex numbers of norm 1 . When $G$ is not abelian, $\widehat{G}$ is not known in general. In 1962, A. Kirillov managed to determine $\widehat{G}$ when $G$ is nilpotent and simply connected [11]: the unitary dual $\widehat{G}$ of $G$ is described by the orbits of the elements of $\mathfrak{g}^{*}$ under the coadjoint action of $G$; this action is defined by the relation

$$
x \cdot l=l \circ \operatorname{Ad}\left(x^{-1}\right), \quad l \in \mathfrak{g}^{*}, x \in G .
$$


From now on, $G$ denotes a simply connected real nilpotent Lie group with Lie algebra $\mathfrak{g}$. For $l$ in $\mathfrak{g}^{*}$, there exists a polarization $\mathfrak{m}$ at $l$, i.e. a subalgebra $\mathfrak{m}$ of $\mathfrak{g}$ which is maximal isotropic for the skew-symmetric bilinear form

$$
B_{l}(X, Y)=l[X, Y], \quad X, Y \in \mathfrak{g} .
$$

Denote by $M$ the connected subgroup exp $\mathfrak{m}$ of $G$ associated to $\mathfrak{m}$. The map

$$
\chi_{l, M}: M \rightarrow U, \quad \exp X \mapsto e^{i\langle l, X\rangle},
$$

is a character of $M$. We write

$$
\pi_{l, M}=\operatorname{ind}_{M}^{G} \chi_{l, M} .
$$

Then $\pi_{l, M}$ is irreducible and the correspondence

$$
\mathfrak{g}^{*} / \operatorname{Ad}^{*}(G) \rightarrow \widehat{G}, \quad[l] \mapsto\left[\pi_{l, M}\right],
$$

is a bijective mapping, called Kirillov's bijection, where

$$
l \sim l^{\prime} \Leftrightarrow \exists x \in G: l^{\prime}=\operatorname{Ad}^{*}(x) l .
$$

The set $\widehat{G}$ is also in bijection with $\operatorname{Prim}(G)$, the space of primitive ideals of the $C^{*}$-algebra of $G$ by [6], and by [3] in bijection with

$\operatorname{Prim}^{*} L^{1}(G)$

$$
=\left\{\operatorname{Ker} \pi|| \pi \text { a } * \text {-topologically irreducible representation of } L^{1}(G)\right\} .
$$

We equip these two sets with the Jacobson topology: for a subset $S$ of $L^{1}(G)$, we define its hull by

$$
h(S)=\left\{J \in \operatorname{Prim}^{*} L^{1}(G)|| S \subset J\right\},
$$

and for a subset $C$ of $\operatorname{Prim}^{*} L^{1}(G)$ or Prim $(G)$, we define its kernel by

$$
k(C)=\bigcap_{J \in C} J
$$

Then, by definition, $C$ is closed in $\operatorname{Prim}^{*} L^{1}(G)$, respectively in $\operatorname{Prim}(G)$, if and only if $C=h(k(C))$. By Brown's theorem [4], Kirillov's bijection is a homeomorphism.

The Jacobson topology is in general not Hausdorff, but always accessible, i.e. each point is closed, which means that every element in $\operatorname{Prim}^{*} L^{1}(G)$, respectively in $\operatorname{Prim}(G)$, is maximal. This follows from the fact that the coadjoint orbits of nilpotent Lie groups are closed [18].

Problem. Given a closed subset $C$ of $\operatorname{Prim}^{*} L^{1}(G)$, can we determine the set $\mathcal{J}(C)$ of closed two-sided ideals of $L^{1}(G)$ with hull $C$ ?

When $\mathcal{J}(C)=\{k(C)\}$, the subset $C$ is said to be of synthesis or spectral. The first result of spectral synthesis is the famous theorem of N. Wiener 
stating that $\emptyset$ is of synthesis in $\operatorname{Prim}^{*} L^{1}(\mathbb{R})$, i.e. each proper closed ideal of $L^{1}(\mathbb{R})$ is contained in the kernel of a $*$-topologically irreducible representation of $L^{1}(\mathbb{R})$. I. Segal [20] next showed that each point of $\operatorname{Prim}^{*} L^{1}(\mathbb{R})$ is of synthesis; then I. Kaplansky [10] generalized this result to $\operatorname{Prim}^{*} L^{1}(G)$ where $G$ is abelian. The first result when $G$ is not abelian was obtained by H. Leptin [12] who showed that if $G$ is nilpotent of step 2, then each point in Prim* $L^{1}(G)$ is of synthesis. If $G$ is nilpotent of step 3, J. Ludwig [14] showed that $\mathcal{J}(\{\operatorname{Ker} \pi\})$ is in bijection with $\mathcal{J}(\{\operatorname{Ker} \chi\})$ where $\chi$ is a character of $L_{w}^{1}\left(\mathbb{R}^{n}\right)$, and $w$ is a weight of polynomial growth on $\mathbb{R}^{n}$. J. Ludwig shows that $\mathcal{J}(\{\operatorname{Ker} \pi\})$ then contains in general an infinity of elements, and consequently $\{\operatorname{Ker} \pi\}$ is not of synthesis in these cases. If $G$ is nilpotent of step 4, the computations become much more difficult and no general result is known. We have however the following theorem due to J. Ludwig [13], which gives the existence of a smallest element in $\mathcal{J}(C)$ :

TheOREM. Let $G$ be a locally compact group with polynomial growth such that $L^{1}(G)$ is symmetric, and $C$ a closed subset of $\operatorname{Prim}^{*} L^{1}(G)$. Then there exists a single closed two-sided ideal $j(C)$ of $L^{1}(G)$ such that

$$
h(j(C))=C
$$

and

$$
\left(J \triangleleft L^{1}(G), h(J) \subset C\right) \Rightarrow j(C) \subset J .
$$

This theorem applies in particular when $G$ is a simply connected nilpotent Lie group [6]. For example, if $G$ is abelian, then $j(C)$ is the closure in $L^{1}(G)$ of the ideal of $L^{1}(G)$ of functions for which the support of the Fourier transform is compact and disjoint from $C$ [19].

Notice that for a closed subset $C$ of $\operatorname{Prim}^{*} L^{1}(G)$, each element of $\mathcal{J}(C)$ is contained in $k(C)$. Hence there exists a "minimal" ideal and a "maximal" ideal with hull $C$. The subset $C$ is then of synthesis if and only if these two ideals are equal.

Let $\pi$ be an element of $\widehat{G}$. In order to determine $\mathcal{J}(\{\operatorname{Ker} \pi\})$ when the step of $G$ is larger than 3 , it is natural to begin with the determination of $j(\{\operatorname{Ker} \pi\})$, since the latter is contained in each element of $\mathcal{J}(\{\operatorname{Ker} \pi\})$. The result obtained by J. Ludwig when $G$ is of step 3 forces us to look for this ideal not in $L^{1}(G)$ but in a weighted $L^{1}$-algebra on $\mathbb{R}^{n}$.

By Kirillov's bijection, $\pi$ is associated to the orbit $O(l)$ of a certain linear form $l$ on $\mathfrak{g}$, and the easiest case is when the orbit $O(l)$ is a single point. The rest of this paper is devoted to the determination of $j(\{\operatorname{Ker} \pi\})$ in this case. This will be done in a quite general class of algebras which contain weighted algebras, and for nilpotent Lie groups of any step. The principal result of this paper is based in fact on a general property of $C^{\infty}(G)$-modules of finite dimension, where $G$ is solvable. This property is dealt with in [2]. 
Notation. By [3], the set $\operatorname{Prim}^{*} L^{1}(G)$ is in bijection with $\mathfrak{g}^{*} / \operatorname{Ad}^{*}(G)$. In order to make the reading easier, closed subsets $C$ of $\operatorname{Prim}^{*} L^{1}(G)$ and closed subsets of $\widehat{G}$ will be identified with closed $\operatorname{Ad}^{*}(G)$-invariant subsets of $\mathfrak{g}^{*}$. So, for $\pi_{l}$ in $\widehat{G}$, associated to the orbit $O(l)$ of a linear form $l$ on $\mathfrak{g}$, the minimal ideal $j\left(\left\{\operatorname{Ker} \pi_{l}\right\}\right)$ of $L^{1}(G)$ and the set $\mathcal{J}\left(\left\{\operatorname{Ker} \pi_{l}\right\}\right)$ of closed two-sided ideals of $L^{1}(G)$ with hull $\left\{\operatorname{Ker} \pi_{l}\right\}$ will be denoted $j(l)$ and $\mathcal{J}(l)$ respectively.

Conventions. Unless otherwise stated, a function will always be complex-valued. For any group, $e$ will indicate the identity element. For a normed algebra $A$ the relation $I \triangleleft A$ means that $I$ is a closed two-sided ideal of $A$.

3. Polynomials and group algebras. In the following, $\lambda$ will indicate a Haar measure on a simply connected nilpotent Lie group $G$ and $d \lambda(x)$ will be denoted by $d x$.

3.1. Notation. Let $G$ be a locally compact group, $\lambda$ a left Haar measure on $G$, and $w$ a weight on $G$. We denote by $L_{w}^{1}(G)$ the subalgebra of $L^{1}(G)$ of measurable functions $f$ such that $\int_{G}|f| w d \lambda$ is finite, and we define a norm \|\|$_{w}$ on $L_{w}^{1}(G)$ by

$$
\|f\|_{w}=\int_{G}|f| w d \lambda
$$

We thus obtain the Beurling algebra $L_{w}^{1}(G)$. The algebra of polynomials on $G$ is denoted by $\mathcal{P}(G)$. For $X$ in $\mathfrak{g}$ and for a $C^{\infty}$ function $f$ on $G$, we let $X * f$ be the left derivative of $f$ in direction $X$, and $f * X$ the right derivative of $f$ in direction $X$ :

$$
\begin{array}{ll}
X * f(y)=\left.\frac{d}{d t} f(\exp (-t X) y)\right|_{t=0}, & y \in G, \\
f * X(y)=\left.\frac{d}{d t} f(y \exp (t X))\right|_{t=0}, & y \in G .
\end{array}
$$

A basis $\left(X_{1}, \ldots, X_{d}\right)$ of $\mathfrak{g}$ being fixed, for a multi-index $\left(\alpha_{1}, \ldots, \alpha_{d}\right)$ of $\mathbb{N}^{d}$, denoted by $\alpha$, and a $C^{\infty}$ function $f$ on $G$, we write

$$
\begin{aligned}
X^{\alpha} * f & =X_{1}^{\alpha_{1}} * \ldots * X_{d}^{\alpha_{d}} * f, \quad f * X^{\alpha}=f * X_{1}^{\alpha_{1}} * \ldots * X_{d}^{\alpha_{d}}, \\
|\alpha| & =\alpha_{1}+\ldots+\alpha_{d} .
\end{aligned}
$$

We denote by $\mathcal{S}(G)$ the Schwartz space of $C^{\infty}$ functions $f$ on $G$ such that for all positive integers $N$,

$$
p_{N}(f)=\sum_{|\alpha| \leq N} \int_{G}\left|X^{\alpha} * f\right| w^{N} d \lambda
$$


is finite, where $w$ is the weight $w_{U}$ defined in 1.3. One can check that the definition of $\mathcal{S}(G)$ is independent of the choice of the basis of $\mathfrak{g}$ and of $U$. We have (see [17])

$$
p_{N}(g * f) \leq p_{N}(g)\|f\|_{w^{N}} .
$$

We denote by $\mathcal{D}(G)$ the subspace of $\mathcal{S}(G)$ of functions with compact support. The space $\mathcal{S}(G)$ equipped with the convolution multiplication and with the family of seminorms $\left(p_{N}\right)_{N \in \mathbb{N}}$ is then a Fréchet algebra and $\mathcal{S}(G)$ is dense in $\left(L^{1}(G),\|\|_{1}\right)$.

3.2. The determination of the "minimal ideal" in Section 5 will be given for a quite general class of algebras. Indeed, in this paper we consider a Banach subalgebra $(A,\|\|)$ of $L^{1}(G)$ containing $\mathcal{S}(G)$ as a dense subspace and satisfying

$$
\left\{\begin{array}{l}
\exists N \in \mathbb{N}, \forall f \in \mathcal{S}(G):\|f\| \leq p_{N}(f), \\
\forall f \in A:\|f\|_{1} \leq\|f\|
\end{array}\right.
$$

which means that the norm \|\| of $A$ makes the injections of $\mathcal{S}(G)$ into $A$ and of $A$ into $L^{1}(G)$ continuous.

3.3. Recall that the characters of $G$, i.e. the continuous homomorphisms of the group $G$ into $\mathbb{C}^{\times}$, are of the form $\exp X \mapsto \chi_{l}(\exp X)=e^{i l(X)}$ where $l$ is an $\mathbb{R}$-linear form on $\mathfrak{g}$ with complex values such that $l[X, Y]$ is zero for all $X$ and $Y$ in $\mathfrak{g}$. For real-valued $l$ we obtain the unitary characters of $G$.

For $l$ in $\mathfrak{g}^{*}$ such that $l$ is zero on $\mathfrak{g}_{1}$, we denote by $\mathcal{P}_{l}$ the vector space of polynomials $P$, with complex coefficients, such that the continuous linear form $P \chi_{l}$ on $\mathcal{S}(G)$ mapping $f$ to $\int_{G} f P \chi_{l} d \lambda$ extends to a continuous linear form on $A$, meaning that there exists a positive number $c$ such that for all $f$ in $\mathcal{S}(G)$, we have

$$
\left|\int_{G} f P \chi_{l} d \lambda\right| \leq c\|f\|
$$

Let $G$ be a group and $s$ be an element of $G$. For a function $f: G \rightarrow \mathbb{C}$, we denote by $L_{s} f$ or ${ }_{s} f$ the left translate of $f$ by $s$, mapping $t$ to $f\left(s^{-1} t\right)$, and by $R_{s} f$ or $f_{s}$ the right translate of $f$ by $s$, mapping $t$ to $f(t s)$.

Let $P$ be in $\mathcal{P}_{l}$ and $f, g$ be elements of $A$. Then $P \chi_{l}$ defines a continuous linear form on $A$ by definition, and consequently $\left\langle P \chi_{l}, g * f\right\rangle$ exists. For $g$ in $A$, we write $\check{g} *\left(P \chi_{l}\right)$ for the continuous linear form on $A$ defined by

$$
\left\langle\check{g} *\left(P \chi_{l}\right), f\right\rangle=\left\langle P \chi_{l}, g * f\right\rangle .
$$

In the same way, $P \chi_{l} * \check{g}$ denotes the continuous linear form on $A$ defined by

$$
\left\langle P \chi_{l} * \check{g}, f\right\rangle=\left\langle P \chi_{l}, f * g\right\rangle .
$$

3.4. Theorem. The vector space $\mathcal{P}_{l}$ is finite-dimensional. 
Proof. 1) Let $f$ be in $\mathcal{S}(G), Q$ a polynomial and $\chi_{q}$ a unitary character of $G$. After an easy computation, for all $x$ in $G$ we have

$$
\left(f *\left(Q \chi_{q}\right)\right)(x)=P(x) \chi_{q}(x)
$$

where $P$ is another polynomial.

2) Let $Q$ be in $\mathcal{P}_{l}$ and $g$ in $\mathcal{S}(G)$. By 1$)$,

$$
g *\left(Q \chi_{l}\right)=Q_{g} \chi_{l}
$$

where $Q_{g}$ is a polynomial, and for all $f$ in $\mathcal{S}(G)$,

$$
\begin{aligned}
\left|\left\langle g *\left(Q \chi_{l}\right), f\right\rangle\right| & =\left|\left\langle Q \chi_{l}, \check{g} * f\right\rangle\right| \leq\left\|Q \chi_{l}\right\|_{\text {op }}\|\check{g} * f\| \\
& \leq\left\|Q \chi_{l}\right\|_{\text {op }} p_{N}(\check{g} * f) \leq\left\|Q \chi_{l}\right\|_{\text {op }} p_{N}(\check{g})\|f\|_{w^{N}}
\end{aligned}
$$

where $N$ is an integer depending on $Q$ and $l$. Hence $g *\left(Q \chi_{l}\right)$ is in the dual space of $L_{w^{N}}^{1}(G)$, and so

$$
\left\|Q_{g} / w^{N}\right\|_{\infty}<\infty
$$

Denote by $\mathcal{P}_{N}$ the vector space of polynomials $P$ such that $\left\|P / w^{N}\right\|_{\infty}$ is finite. Since the weight $w^{N}$ has a polynomial growth, the space $\mathcal{P}_{N}$ is finitedimensional and we have shown that for all $Q$ in $\mathcal{P}_{l}$ and all $g$ in $\mathcal{S}(G)$, $g *\left(Q \chi_{l}\right)$ belongs to $\mathcal{P}_{N} \chi_{l} \cap \mathcal{P}_{l} \chi_{l}$.

3) Let $Q$ be in $\mathcal{P}_{l}$. Since the weak star topology on $\mathcal{P}_{N}$ with respect to $L_{w^{N}}^{1}(G)$ coincides with the norm topology, and since for any approximate identity $\left(g_{n}\right)$ in $\mathcal{S}(G),\left(g_{n} * Q \chi_{l}\right)$ converges in the weak star topology to $Q \chi_{l}$, it follows that $\left(g_{n} * Q \chi_{l}\right)$ inside $\mathcal{P}_{N}$ converges to $Q \chi_{l}$ in the operator norm, and so $Q \chi_{l} \in \mathcal{P}_{N}$. Hence $\mathcal{P}_{l} \subset \mathcal{P}_{N}$.

3.5. Notation. Until the end of this paper, $W$ indicates a nonzero subspace of $\mathcal{P}_{l}$ which is invariant under left and right translations, and $W \chi_{l}$ is denoted by $W_{l}$. We also write

$$
I(W)=\left\{f \in A \mid \forall P \in W:\left\langle P \chi_{l}, f\right\rangle=0\right\}=\left(W \chi_{l}\right)^{\circ} .
$$

We then have the following proposition.

3.6. Proposition. The vector space $W$ is invariant under translations and under convolution by elements of $\mathcal{S}(G)$. So $I(W)$ is a closed two-sided ideal of $A$.

\section{Hull}

Definition. For a Banach algebra $A$, we denote by $\operatorname{Prim}(A)$ the set of primitive ideals of $A$, i.e. the set of the kernels of algebraically irreducible representations of $A$ in Banach spaces. The kernel of a subset $C$ of $\operatorname{Prim}(A)$ is the set

$$
k(C)=\bigcap_{J \in C} J
$$


and the hull of a subset $S$ of $A$ is the set

$$
h(S)=\{J \in \operatorname{Prim}(A) \mid S \subset J\} .
$$

Notation. For a Banach algebra $A$, the set $\operatorname{Prim}(A)$ is equipped with the Jacobson topology: by definition, a subset $C$ of $\operatorname{Prim}(A)$ is closed in $\operatorname{Prim}(A)$ if and only if $C=h(k(C))$. We denote by $\mathcal{J}(C)$ the set of closed two-sided ideals of $A$ with hull $C$ :

$$
\mathcal{J}(C)=\{J \triangleleft A \mid h(J)=C\} .
$$

In the present case, the set $\left\{\operatorname{Ker} \chi_{l}\right\}$ is closed in $\operatorname{Prim}(A)$, and as stipulated in Section 2, the set $\mathcal{J}\left(\left\{\operatorname{Ker} \chi_{l}\right\}\right)$ will be denoted $\mathcal{J}(l)$ by abuse of notation.

4.1. Proposition. With the above hypothesis on A, we have

$$
\operatorname{Prim}(A)=\left\{\operatorname{Ker}\left(\left.\pi\right|_{A}\right) \mid \pi \in \widehat{G}\right\} .
$$

Proof. 1) Let $\pi$ be a unitary topologically irreducible representation of $G$; denote also by $\pi$ the corresponding representation of $L^{1}(G)$. Since $A$ is dense in $L^{1}(G),\left.\pi\right|_{A}$ is topologically irreducible on the Hilbert space $\mathcal{H}$. Let

$$
\mathcal{H}_{0}=\operatorname{Span}\{\pi(f) \xi \mid \xi \in \mathcal{H}, f \in A, \pi(f) \text { of finite rank }\} .
$$

Since $\pi(\mathcal{S}(G))$ contains many operators of finite rank, $\mathcal{H}_{0}$ is an $A$-invariant nontrivial subspace of $\mathcal{H}$ and the restriction of $\pi$ to $\mathcal{H}_{0}$ defines a simple module of $A$ (see [6]). Hence $\operatorname{Ker}\left(\left.\pi\right|_{A}\right)$ is a primitive ideal:

$$
\left\{\operatorname{Ker}\left(\left.\pi\right|_{A}\right) \mid \pi \in \widehat{G}\right\} \subset \operatorname{Prim}(A) .
$$

Let us prove the other inclusion. If $(T, V)$ is a simple $A$-module on a Banach space $V$ then $\left(\left.T\right|_{\mathcal{S}(G)}, V\right)$ is a topologically irreducible $\mathcal{S}(G)$-module. Hence by [16] there exists a $\pi \in \widehat{G}$ such that

$$
\operatorname{Ker}\left(\left.T\right|_{\mathcal{S}(G)}\right)=\operatorname{Ker}\left(\left.\pi\right|_{\mathcal{S}(G)}\right) \text {. }
$$

By [15] we know that $\operatorname{Ker}\left(\left.\pi\right|_{\mathcal{S}(G)}\right)$ is dense in $\operatorname{Ker}\left(\left.\pi\right|_{A}\right)$. Hence $\operatorname{Ker} T$ contains $\operatorname{Ker}\left(\left.\pi\right|_{A}\right)$.

2) Let us prove that $\operatorname{Ker}\left(\left.\pi\right|_{A}\right)$ is a maximal two-sided ideal of $A$. Let $M$ be a closed two-sided ideal of $A$ containing $\operatorname{Ker}\left(\left.\pi\right|_{A}\right)$. Suppose that $M \neq$ $\operatorname{Ker}\left(\left.\pi\right|_{A}\right)$. Then there exists $g$ in $M$ such that $g \notin \operatorname{Ker}\left(\left.\pi\right|_{A}\right)$. By [15], the two-sided ideal

$$
R=\{f \in \mathcal{S}(G) \mid \pi(f) \text { of finite rank }\}
$$

is dense in $\mathcal{S}(G)$ and then in $A$. Hence $R * g * R$ is not contained in $\operatorname{Ker}\left(\left.\pi\right|_{A}\right)$ and so $M$ contains an element $h$ such that $\pi(h)=P_{\lambda}$ is the orthogonal projector onto a $C^{\infty}$ vector $\lambda$ of $\mathcal{H}_{\pi}$. Let $f$ in $\mathcal{S}(G)$ be such that $\pi(f)=P_{\mu}$ is also a one-dimensional orthogonal projector with $\langle\lambda, \mu\rangle \neq 0$. Then

$$
\pi(f)=|\langle\lambda, \mu\rangle|^{-2} P_{\mu} \circ P_{\lambda} \circ P_{\mu}=\pi\left(\langle\lambda, \mu\rangle^{-2} f * h * f\right) .
$$

Hence 


$$
f-\langle\lambda, \mu\rangle^{-2} f * h * f \in \operatorname{Ker} \pi \subset M
$$

and consequently $f \in M$. Since $R$ is generated as an ideal by those elements $f$, this shows that $M$ contains the ideal $R$ and finally $M=A$ since $M$ is closed. This proves that $\operatorname{Ker} T=\operatorname{Ker}\left(\left.\pi\right|_{A}\right)$.

The aim of this section is to determine the hull of $I(W)$ where $W$ is defined in 3.5. Since $W$ is finite-dimensional, we have the following proposition.

4.2. Proposition. The space $W$ is invariant under derivations: for all $X$ in $\mathfrak{g}$ and all $P$ in $W, X * P$ and $P * X$ belong to $W$.

By [5], we have:

4.3. Proposition. There exists a function deg on the complex vector space of polynomials on $G$ such that for all $X$ in $\mathfrak{g}$ and all polynomials $P$, we have

$$
\operatorname{deg}(X * P)<\operatorname{deg} P .
$$

Hence for all $X$ in $\mathfrak{g}$, there exists a natural $k$ such that for all $P$ in $W$, $X^{k} * P$ is zero.

4.4. Proposition. The hull $h(I(W))$ of $I(W)$ contains Ker $\chi_{l}$.

Proof. For $X$ in $\mathfrak{g}$ and $P$ in $W, \pi(X)\left(P \chi_{l}\right)=X *\left(P \chi_{l}\right)=(X * P) \chi_{l}+$ $i\langle l, X\rangle\left(P \chi_{l}\right)$ defines a representation $\pi$ of the Lie algebra $\mathfrak{g}$ in $W_{l}$. By Lie's theorem (see [7]), there exists a nonzero element $P$ in $W$ such that for all $X$ in $\mathfrak{g}, \pi(X)\left(P \chi_{l}\right)=\lambda(X)\left(P \chi_{l}\right)$ where $\lambda$ is a linear form on $\mathfrak{g}$. Since $\operatorname{deg}(X * P)<\operatorname{deg} P$, we have $\lambda(X)=i\langle l, X\rangle$ and so $(X * P) \chi_{l}=0$. Hence $X * P=0$ and the polynomial $P$ is constant. Consequently, $\chi_{l} \in W_{l}$ and hence $I(W) \subset \operatorname{Ker} \chi_{l}$ and Ker $\chi_{l} \subset h(I(W))$.

Notation. For $f$ in $L^{1}(G)$, the Fourier transform of $f$ at $l$ is denoted $\widehat{f}(l)$ and is defined by

$$
\widehat{f}(l)=\int_{G} f \bar{\chi}_{l} d \lambda .
$$

Let $P$ be a polynomial in the variables $X_{1}, \ldots, X_{d}$. We define the differential operator $P(D)$ in the $D_{j}=i \partial / \partial X_{j}$ with

$$
D^{\alpha}=\prod_{i=1}^{d} D_{j}^{\alpha_{j}}, \quad \alpha=\left(\alpha_{1}, \ldots, \alpha_{d}\right) .
$$

We have the well known result:

4.5. Lemma. For all $f$ in $A$,

$$
f \in I(W) \Leftrightarrow \forall P \in W:(P(D)(\widehat{f}))(-l)=0,
$$

where $\widehat{f}$ indicates the Fourier transform of $f$.

4.6. Theorem. The hull $h(I(W))$ of $I(W)$ is $\left\{\operatorname{Ker} \chi_{l}\right\}$. 
Proof. By Proposition 4.4, Ker $\chi_{l} \in h(I(W))$.

Let $\pi$ be a topologically irreducible $*$-representation of $L^{1}(G)$ in a Hilbert space whose kernel in $A$ contains $I(W)$. By Theorem 3.4, $I(W)$ is of finite codimension in $A$, hence $\pi$ is finite-dimensional and defines an irreducible continuous unitary representation $\widetilde{\pi}$ of the nilpotent group $G$. By Lie's theorem (see [7]), $\widetilde{\pi}$ is a character. Then $\pi$ is a character $\chi_{l^{\prime}}$ where $l^{\prime}$ is a real linear form on $\mathfrak{g}$ which is zero on $[\mathfrak{g}, \mathfrak{g}]$ by 3.3. If $l^{\prime}$ is different from $l$, there exists $f$ in $\mathcal{S}(\mathfrak{g})$ such that $\widehat{f}\left(-l^{\prime}\right)=1$ and $\widehat{f}$ is zero on a neighborhood of $-l$. Then $f$ does not belong to Ker $\chi_{l^{\prime}}$ and belongs to $I(W)$ by Lemma 4.5. Since this contradicts the hypothesis, $l^{\prime}$ is equal to $l$.

\section{Minimal ideal}

5.1. Proposition. For each closed subset $C$ of $\operatorname{Prim}^{*}(A)$, there exists a closed two-sided ideal $j(C)$ of $A$ with hull $C$ such that each closed two-sided ideal of $A$ whose hull is contained in $C$ contains $j(C)$.

Proof. The proof given in [13] adapts to the general case.

Taking in the previous theorem $W=\mathcal{P}_{l}$, we have $j\left(\operatorname{Ker} \chi_{l}\right) \subset I\left(\mathcal{P}_{l}\right)$. The following theorem will show the other inclusion.

5.2. Lemma. Let $F$ be a finite-dimensional A-left invariant subspace of the dual $A^{\prime}$ of the algebra $A$. Then each element of $F$ is a finite sum of functions of the form $P \chi_{q}$, where $P$ is a polynomial, and $\chi_{q}$ a unitary character of $G$.

Proof. Let us show that the elements of $F$ are $C^{\infty}$ functions on $G$. Let $\left(\mu_{1}, \ldots, \mu_{n}\right)$ be a basis of $F$. Then $\mathcal{D}(G) * \mu_{1}+\ldots+\mathcal{D}(G) * \mu_{n}$ is dense in the finite-dimensional vector space $F$, hence is equal to $F$. Every $\mu$ in $F$ defines a tempered distribution on $G$. Let $g$ be in $\mathcal{D}(G)$. For all $f$ in $\mathcal{S}(G)$,

$$
\langle g * \mu, f\rangle=\langle\mu, \check{g} * f\rangle=\int_{G} \varphi(x)(1-\Delta)^{N}(\check{g} * f)(x) d x
$$

for a certain function $\varphi$ with moderate growth, of class $C^{\infty}$ on $G$, and a certain integer $N$, where $\Delta$ indicates the Laplacian of $G$ (by [17]).

Putting $h=(1-\Delta)^{N} \check{g}$, we then have

$$
\langle g * \mu, f\rangle=\int_{G} \psi f d \lambda \quad \text { where } \quad \psi(x)=\int_{G} h \varphi_{x} d \lambda .
$$

The linear form $g * \mu$ is then given on $\mathcal{S}(G)$ by a function $\psi$ of class $C^{\infty}$ on $G$. Since $\mathcal{S}(G)$ is dense in $A$, the linear form $g * \mu$ can be identified with $\psi$, and with this identification, $F$ consists of $C^{\infty}$ functions. The lemma then results from Proposition 1 of [2]. 
5.3. Theorem. The smallest closed two-sided ideal of $A$ with hull $\left\{\operatorname{Ker} \chi_{l}\right\}$ is

$$
j(l)=I\left(\mathcal{P}_{l}\right) .
$$

Proof. 1) It has already been noticed that $j(l)$ is contained in $I\left(\mathcal{P}_{l}\right)$. By [15], there exists a natural integer $N$ such that $j(l)=\overline{\left(\operatorname{Ker} \chi_{l}\right)^{N}}$.

Let us show by induction on $n$ that if $T$ is a continuous linear form on $A$ which is zero on $\left(\operatorname{Ker} \chi_{l}\right)^{n}$ then $T$ is of the form $P \chi_{l}$ where $P$ belongs to $\mathcal{P}_{l}$.

The result is true if $n=1$ : the polynomial $P$ is a nonzero constant.

2 ) Let $m$ in $\mathbb{N}^{*}$ be such that $T$ is zero on $\left(\operatorname{Ker} \chi_{l}\right)^{m}$ and nonzero on $\left(\text { Ker } \chi_{l}\right)^{m-1}$.

(a) Let $f_{0}$ be in Ker $\chi_{l}$. Then $\check{f}_{0} * T$ is a continuous linear form on $A$ and for all $u$ in $\left(\operatorname{Ker} \chi_{l}\right)^{m-1}$,

$$
\left\langle\check{f}_{0} * T, u\right\rangle:=\left\langle T, f_{0} * u\right\rangle=0
$$

because $f_{0} * u$ belongs to $\left(\operatorname{Ker} \chi_{l}\right)^{m}$. The induction hypothesis shows that $\check{f}_{0} * T=P_{f_{0}} \chi_{l}$ where $P_{f_{0}}$ belongs to $\mathcal{P}_{l}$.

(b) Let $f$ and $f_{1}$ in $A$ be such that $\chi_{l}\left(f_{1}\right)=1$. Then $f-\chi_{l}(f) f_{1} \in \operatorname{Ker} \chi_{l}$, and consequently

$$
\left(f-\widehat{f}(-l) f_{1}\right)^{\vee} * T=P_{f} \chi_{l}
$$

where $P_{f} \in \mathcal{P}_{l}$ by (a), i.e.

$$
\check{f} * T=\widehat{f}(-l) \check{f}_{1} * T+P_{f} \chi_{l} \in \mathbb{C}\left(\check{f}_{1} * T\right)+\mathcal{P}_{l} \chi_{l} .
$$

This shows that the complex vector space $\check{A} * T$, which is contained in $A^{\prime}$, is of finite dimension by Theorem 3.4.

3) Let $\phi$ be an element of $A$. By 2) and Lemma $5.2, \check{\phi} * T$ is of the form

$$
\check{\phi} * T=\sum_{j=1}^{p} P_{j} \chi_{q_{j}}
$$

where the $P_{j}$ are polynomials and the $\chi_{q_{j}}$ are unitary characters of $G$ which we assume to be all distinct. Let us show that $p=1$ and $q_{1}=l$.

Let $f_{0}$ be in $\operatorname{Ker} \chi_{l} \cap \mathcal{S}(G)$. The function $f_{0} * \phi$ belongs to Ker $\chi_{l}$, so by 2$)(\mathrm{a})$,

$$
\left(f_{0} * \phi\right)^{\vee} * T=P \chi_{l}
$$

where $P$ belongs to $\mathcal{P}_{l}$. On the other hand, the computation 1 ) in the proof of Theorem 3.4 shows that

$$
\left(f_{0} * \phi\right)^{\vee} * T=\sum_{j=1}^{p} \check{f}_{0} * P_{j} \chi_{q_{j}}=\sum_{j=1}^{p} Q_{j} \chi_{q_{j}}
$$

where the $Q_{j}$ are polynomials which we can assume to be all nonzero. Finally

$$
P \chi_{l}=\sum_{j=1}^{p} Q_{j} \chi_{q_{j}} .
$$


In the module of linear combinations (whose coefficients are polynomials) of unitary characters of $G$, each finite family of distinct unitary characters of $G$ is free. Consequently, $p=1, q_{1}=l$ and $\tilde{\phi} * T=Q \chi_{l}$ where $Q$ is a polynomial. Since $\phi \in A$ and $T \in A^{\prime}, \check{\phi} * T$ is continuous on $A$ and $Q$ belongs to $\mathcal{P}_{l}$.

Let us show that $T$ itself is in $\mathcal{P}_{l} \chi_{l}$.

4) The space $\mathcal{P}_{l}$ being finite-dimensional, let $f_{1}, \ldots, f_{M}$ be Schwartz functions on $G$ such that

$$
\left(\left\langle P \chi_{l}, f_{i}\right\rangle=0 \text { for } i=1, \ldots, M\right) \Rightarrow P=0 .
$$

For all $P$ in $\mathcal{P}_{l}$ let

$$
\left\|P \chi_{l}\right\|_{l}=\max _{1 \leq i \leq M}\left|\left\langle P \chi_{l}, f_{i}\right\rangle\right| .
$$

Let $\left(\phi_{n}\right)_{n \in \mathbb{N}}$ be an approximate unit in $\mathcal{S}(G)$. For all $f$ in $\mathcal{S}(G)$,

$$
\left\langle\check{\phi}_{n} * T-T, f\right\rangle=\left\langle T, \phi_{n} * f-f\right\rangle .
$$

The sequence $\left(\phi_{n} * f-f\right)_{n \in \mathbb{N}}$ converges to 0 in $\mathcal{S}(G)$, hence in $A$, and $T$ being continuous on $A,\left(\left\langle\dot{\phi}_{n} * T-T, f\right\rangle\right)_{n \in \mathbb{N}}$ tends to 0 by (1). We have

$$
\left\|\check{\phi}_{n} * T-\check{\phi}_{m} * T\right\|_{l}=\max _{1 \leq i \leq M}\left|\left\langle T,\left(\phi_{n}-\phi_{m}\right) * f_{i}\right\rangle\right| .
$$

This tends to 0 because $\left(\phi_{n}-\phi_{n} * f_{i}\right)_{n \in \mathbb{N}}$ tends to 0 in $\mathcal{S}(G)$, hence also in $A$. This shows that the sequence $\left(\check{\phi}_{n} * T\right)_{n \in \mathbb{N}}$ is Cauchy for the norm \|\|$_{l}$, hence converges to an element $P \chi_{l}$ where $P$ belongs to $\mathcal{P}_{l}$, the space $\mathcal{P}_{l} \chi_{l}$ being finite-dimensional. Let $f$ be in $\mathcal{S}(G)$. For all $Q$ in $\mathcal{P}_{l}$ write

$$
\left\|Q \chi_{l}\right\|_{f}=\left\|Q \chi_{l}\right\|_{l}+\left|\left\langle Q \chi_{l}, f\right\rangle\right| \text {. }
$$

Then \|\|$_{f}$ is a norm on $\mathcal{P}_{l} \chi_{l}$ equivalent to \|\|$_{l}$, since $\mathcal{P}_{l} \chi_{l}$ is finite-dimensional. Hence the sequence $\left(\check{\phi}_{n} * T\right)_{n \in \mathbb{N}}$ converges to $P \chi_{l}$ for \|\|$_{f}$ and the inequality

$$
\begin{aligned}
\left|\left\langle P \chi_{l}-T, f\right\rangle\right| & \leq\left|\left\langle P \chi_{l}-\check{\phi}_{n} * T, f\right\rangle\right|+\left|\left\langle\check{\phi}_{n} * T-T, f\right\rangle\right| \\
& \leq\left\|P \chi_{l}-\check{\phi}_{n} * T\right\|_{f}+\left|\left\langle\check{\phi}_{n} * T-T, f\right\rangle\right|,
\end{aligned}
$$

valid for all $n$ in $\mathbb{N}$, gives, as $n \rightarrow \infty$,

$$
\left\langle P \chi_{l}-T, f\right\rangle=0 .
$$

Since $\mathcal{S}(G)$ is dense in $A$, this proves that $T=P \chi_{l}$ and so $T$ is zero on $I\left(\mathcal{P}_{l}\right)$. For all $T$ in $j(l)^{\circ}$, we know that $T$ is zero on $\left(\operatorname{Ker} \chi_{l}\right)^{N}$ and by the preceding $T$ belongs to $\mathcal{P}_{l} \chi_{l}$ and so to $I\left(\mathcal{P}_{l}\right)^{\circ}$. Since $\left\langle T,\left(\operatorname{Ker} \chi_{l}\right)^{m}\right\rangle=0$ we see that $T$ is zero on $I\left(\mathcal{P}_{l}\right)$. The Hahn-Banach theorem shows finally that $I\left(\mathcal{P}_{l}\right)$ is contained in $j(l)$.

Notation. Let $J$ be a closed two-sided ideal of $A$. We associate to it the vector subspace $V(J)$ of $\mathcal{P}_{l}$ defined by

$$
V(J)=\left\{P \in \mathcal{P}_{l} \mid \forall f \in J: P f \in \operatorname{Ker} \chi_{l}\right\} .
$$


We show that the mapping $J \mapsto V(J)$ gives characterization of the closed two-sided ideals of $A$ with hull $\left\{\operatorname{Ker} \chi_{l}\right\}$.

5.4. Proposition. Let $J$ be a closed two-sided ideal of $A$. The vector subspace $V(J)$ of $\mathcal{P}_{l}$ is invariant under translations.

Proof. The vector space generated by $\mathcal{S}(G) * V(J) * \mathcal{S}(G)$ is dense in the finite-dimensional vector space $V(J)$, hence is equal to $V(J)$. The result then follows from the formula

$$
{ }_{x}(f * P * g)_{y}={ }_{x} f * P * g_{y}
$$

valid for all $f$ and $g$ in $\mathcal{S}(G), P$ in $V(J)$, and $x, y$ in $G$.

Notation. Denote by $\mathcal{T} \mathcal{P}_{l}$ the set of nonzero subspaces of $\mathcal{P}_{l}$ which are invariant under left and right translations. For a topological vector space $E$ and a subset $X$ of $E$, we denote by $X^{\circ}$ the orthogonal complement of $X$ in $E$, i.e. the vector space of continuous linear forms on $E$ which are zero on $X$ :

$$
X^{\circ}=\left\{\varphi \in E^{\prime} \mid \forall x \in X:\langle\varphi, x\rangle=0\right\} .
$$

The most important result of this paper is the following theorem:

5.5. Theorem. The map

$$
\mathcal{T} \mathcal{P}_{l} \rightarrow \mathcal{J}(l), \quad W \mapsto I(W),
$$

is a decreasing bijection, with inverse

$$
\mathcal{J}(l) \rightarrow \mathcal{T} \mathcal{P}_{l}, \quad J \mapsto V(J) .
$$

Proof. By Theorem 4.6, the map $W \mapsto I(W)$ is $\mathcal{J}(l)$-valued.

For any finite-dimensional subspace $U$ of $A^{\prime}$, we know that $U$ is $*$-weakly closed and so $\left(U^{\circ}\right)^{\circ}=U$. This shows that the mapping $W \mapsto I(W)$ is injective.

Let us show the surjectivity. Let $J$ be an element of $\mathcal{J}(l)$. Since $J \supset j(l)$, its orthogonal $J^{\circ}$ is finite-dimensional and is contained in $j(l)^{\circ}$, which means by Theorem 5.3 that $J^{\circ} \subset \mathcal{P}_{l} \chi_{l}$ and so $J^{\circ}=W \chi_{l}$ for some translation invariant subspace $W$ of $\mathcal{P}_{l}$. Hence $J=\left(W \chi_{l}\right)^{\circ}=I(W)$, which shows the surjectivity of the map $W \mapsto I(W)$ and consequently, the bijectivity of $J \mapsto V(J)$.

6. Examples. Let $w$ be a symmetric weight with polynomial growth on $G$. Let $N$ be an integer and define $A_{N}$ as the subalgebra of $L^{1}(G)$ of classes of functions $f$ such that $\sum_{|\alpha| \leq N} \int_{G}\left(\left|X^{\alpha} * f\right| w+\left|f * X^{\alpha}\right| w\right) d \lambda$ is finite. We define a norm on $A_{N}$ by putting

$$
\|f\|=\sum_{|\alpha| \leq N} \int_{G}\left(\left|X^{\alpha} * f\right| w+\left|f * X^{\alpha}\right| w\right) d \lambda .
$$


The algebra $A_{N}$ with the norm \|\| is a Banach algebra and satisfies the conditions given in 3.2. Consequently, Theorem 5.3 applies in this case. In particular, for $N$ equal to zero, the weighted algebra $L_{w}^{1}(G)$ defined in 3.1 is an example of an algebra $A$ satisfying the conditions of 3.2. The rest of this section states the principal results of the paper in this particular case.

Notation. Let $G$ be a locally compact group and $w$ a weight on $G$. We denote by $L_{w}^{\infty}(G)$ the vector space of (classes of) functions $f$ essentially bounded by $w$, i.e. such that $\|f / w\|_{\infty}$ is finite, and we define a norm \|\| on $L_{w}^{\infty}(G)$ by

$$
\|f\|=\|f / w\|_{\infty} .
$$

The following proposition, which describes the topological dual $L_{w}^{1}(G)^{\prime}$ of $L_{w}^{1}(G)$, is known.

6.1. Proposition. Let $G$ be a locally compact group, $\lambda$ a nonzero positive left Haar measure on $G$, and $w$ a weight on $G$. The map $\psi: L_{w}^{\infty}(G) \rightarrow$ $L_{w}^{1}(G)^{\prime}$ which takes $g \in L_{w}^{\infty}(G)$ to

$$
\psi g: L_{w}^{1}(G) \rightarrow \mathbb{C}, \quad f \mapsto\langle g, f\rangle=\int_{G} f g d \lambda,
$$

is an isometric isomorphism of Banach spaces.

In the following, the spaces $L_{w}^{1}(G)^{\prime}$ and $L_{w}^{\infty}(G)$ will be identified. The topological dual of $L_{w}^{1}(G)$ being known, it is possible to give a more vivid description of the vector space $\mathcal{P}_{l}$ defined in 3.3:

Notation. Let $w$ be a weight on $G$. We denote by $\mathcal{P}_{w}(G)$ the vector space of polynomials which are essentially bounded by $w$ :

$$
\mathcal{P}_{w}(G)=\mathcal{P}(G) \cap L_{w}^{\infty}(G) .
$$

By 6.1, it is clear that $\mathcal{P}_{l}=\mathcal{P}_{w}(G)$ and Theorem 5.3 can be written as

$$
j(l)=I\left(\mathcal{P}_{w}(G)\right)=\left\{f \in L_{w}^{1}(G) \mid \forall P \in \mathcal{P}_{w}(G): \int_{G} P(x) f(x) \chi_{l}(x) d x=0\right\} .
$$

Particular cases. 1) If $w$ is the constant weight equal to 1 , then $L_{w}^{1}(G)$ coincides with $L^{1}(G)$, and $\mathcal{P}_{w}(G)$ contains only constants; then $j(l)=$ $\left\{\operatorname{Ker} \chi_{l}\right\}$, which shows that $\left\{\operatorname{Ker} \chi_{l}\right\}$ is of synthesis. We find again in this case a result of $[10]$.

2) If in a direction $X_{0}, w\left(\exp \left(t X_{0}\right)\right)$ grows at least as $|t|$, then $\mathcal{P}_{w}(G)$ contains a nonconstant polynomial, hence $j(l)$ is strictly contained in $\left\{\operatorname{Ker} \chi_{l}\right\}$ and therefore $\left\{\operatorname{Ker} \chi_{l}\right\}$ is not of synthesis. So, for a nonconstant weight $w$, the one-point set $\left\{\operatorname{Ker} \chi_{l}\right\}$ is not of synthesis in $L_{w}^{1}(G)$ in general. 
6.2. Let us take for $G$ the 3 -dimensional Heisenberg group $H_{1}$, for which the multiplication is given by

$$
(x, y, z) \cdot\left(x^{\prime}, y^{\prime}, z^{\prime}\right)=\left(x+x^{\prime}, y+y^{\prime}, z+z^{\prime}+\frac{1}{2}\left(x y^{\prime}-y x^{\prime}\right)\right) .
$$

Denote by $L$ the left regular representation of $H_{1}$ in $C^{\infty}\left(H_{1}\right)$. Let $P$ be the polynomial

$$
P=-x^{2}+y^{2}+z^{2}
$$

and $V$ the vector space generated by $P$ and its left derivatives. Then the vector space $V$ is 10 -dimensional and $\left(1, x, y, z, x^{2}, y^{2}, x y, x z, y z, z^{2}\right)$ is a basis of $V$. For an element $\left(m_{i j}\right)_{1 \leq i, j \leq 10}$, denoted by $M$, belonging to $\operatorname{End}(V)$, denote by $\|M\|_{\text {HS }}$ its Hilbert-Schmidt norm

$$
\|M\|_{\mathrm{HS}}=\left(\sum_{1 \leq i, j \leq 10}\left|m_{i j}\right|^{2}\right)^{1 / 2} .
$$

Finally, define

$$
\omega(u, v, w)=\left\|L_{(u, v, w)}\right\|_{\mathrm{HS}} .
$$

An explicit computation shows that

$$
\begin{aligned}
\omega(u, v, w)= & {\left[10+\frac{35}{4}\left(u^{2}+v^{2}\right)+7 w^{2}+\frac{7}{4} u^{2} v^{2}\right.} \\
& \left.+2\left(u^{2} w^{2}+v^{2} w^{2}\right)+\frac{21}{16}\left(u^{4}+v^{4}\right)+w^{4}\right]^{1 / 2} .
\end{aligned}
$$

The mapping $\omega$ is a weight on $H_{1}$. Let $\pi$ be an element of the unitary dual $\widehat{H}_{1}$ of $H_{1}$ and let $\mathfrak{h}_{1}$ be the Lie algebra of $H_{1}$. Assume that the orbit of the linear form $l$ on $\mathfrak{h}_{1}$ associated to $\pi$ by the Kirillov bijection is one point, i.e. $l$ is a character of $\mathfrak{h}_{1}$. So, $\pi$ is a character of $L_{\omega}^{1}\left(H_{1}\right)$. By Theorem 5.5, the sets $\mathcal{J}(l)$ and $\mathcal{T} \mathcal{P}_{\omega}\left(H_{1}\right)$ are in bijection. The set $\mathcal{T} \mathcal{P}_{\omega}\left(H_{1}\right)$ is explicitly determined in $[1]$.

\section{References}

[1] D. Alexander, Idéaux minimaux d'algèbres de groupes, thèse, Université de Metz, 2000 .

[2] D. Alexander and J. Ludwig, Undecomposable finite dimensional representations of solvable Lie groups, preprint.

[3] J. Boidol, H. Leptin, J. Schurmann und D. Vahle, Raüme primitiver Ideale von Gruppenalgebren, Math. Ann. 236 (1978), 1-13.

[4] I. D. Brown, Dual topology of a nilpotent Lie group, Ann. Sci. École Norm. Sup. 6 (1973), 407-411.

[5] S. Dhieb et J. Ludwig, Caractérisation des convoluteurs de Schwartz des groupes de Lie nilpotents, J. Funct. Anal. 144 (1997), 46-53. 
[6] J. Dixmier, Opérateurs de rang fini dans les représentations unitaires, Inst. Hautes Études Sci. Publ. Math. 6 (1960), 13-35.

[7] —, Algèbres enveloppantes, Cahiers Sci. 37, Gauthier-Villars, 1974.

[8] E. Hille and R. S. Phillips, Functional Analysis and Semi-Groups, Amer. Math. Soc., Providence, 1957.

[9] A. Hulanicki, Subalgebra of $L_{1}(G)$ associated with Laplacian on a Lie group, Colloq. Math. 31 (1974), 259-287.

[10] I. Kaplansky, Primary ideals in group algebras, Proc. Natl. Acad. Sci. USA 35 (1949), 133-136.

[11] A. Kirillov, Unitary representations of nilpotent Lie groups, Uspekhi Mat. Nauk 17 (1962), no. 4, 57-110 (in Russian); English transl.: Russian Math. Surveys 17 (1962), no. 4, 53-104.

[12] H. Leptin, Ideal theory in group algebras of locally compact groups, Invent. Math. 31 (1976), 259-278.

[13] J. Ludwig, Polynomial growth and ideals in group algebras, Manuscripta Math. 30 (1980), 215-221.

[14] —, On the spectral synthesis problem for points in the dual of a nilpotent Lie group, Ark. Math. 21 (1983), 127-144.

[15] - Minimal $C^{*}$-dense ideals and algebraically irreducible representations in the Schwartz algebra of a nilpotent Lie group, in: Lecture Notes in Math. 1359, Springer, Berlin, 1988, 209-217.

[16] - , Topologically irreducible representations of the Schwartz algebra of a nilpotent Lie group, Arch. Math. (Basel) 54 (1990), 284-292.

[17] J. Ludwig and C. Molitor-Braun, A restriction theorem for ideals in the Schwartz algebra of a nilpotent Lie group, ibid. 67 (1996), 199-210.

[18] L. Pukanszky, Leçons sur les représentations des groupes, Dunod, 1967.

[19] H. Reiter, Classical Harmonic Analysis and Locally Compact Groups, Clarendon Press, 1968.

[20] I. Segal, The group algebra of a locally compact group, Trans. Amer. Math. Soc. 61 (1947), 69-105.

[21] N. Varopoulos, Analysis and Geometry on Groups, Cambridge Univ. Press, 1992.

Département de Mathématiques et d'Informatique

Université de Metz

Ile du Saulcy

57045 Metz Cedex 01, France

E-mail: alexander@poncelet.sciences.univ-metz.fr

ludwig@poncelet.sciences.univ-metz.fr 